\title{
Construction of admissible linear orders for interval-valued Atanassov intuitionistic fuzzy sets with an application to decision making
}

\author{
L. De Miguel ${ }^{\mathrm{a}}$, H. Bustince ${ }^{\mathrm{a}, \mathrm{c}}$, J. Fernandez $^{\mathrm{a}}$, E. Induráin ${ }^{\mathrm{b}}$, A. Kolesárovád ${ }^{\mathrm{d}}$, R. Mesiar ${ }^{\mathrm{d}, \mathrm{e}}$ \\ ${ }^{a}$ Departamento de Automática y Computación, Universidad Pública de Navarra, \\ Campus Arrosadia s/n, 31006, Pamplona, Spain \\ ${ }^{b}$ Departamento de Matemáticas, Universidad Pública de Navarra, \\ Campus Arrosadia s/n, 31006, Pamplona, Spain \\ ${ }^{c}$ Institute of Smart Cities, Universidad Publica de Navarra, \\ Campus Arrosadia s/n, 31006 Pamplona, Spain \\ ${ }^{d}$ Institute of Information Engineering, Automation and Mathematics, \\ Slovak University of Technology, 81237 Bratislava, Slovakia \\ ${ }^{e}$ Slovak University of Technology, Radlinskeho 11, Bratislava, Slovakia \\ Institute of Information Theory and Automation, \\ Academy of Sciences of the Czech Republic, 18208 Prague, Czech Republic
}

\begin{abstract}
In this work we introduce a method for constructing linear orders between pairs of intervals by using aggregation functions. We adapt this method to the case of interval-valued Atanassov intuitionistic fuzzy sets and we apply these sets and the considered orders to a decision making problem.
\end{abstract}

Keywords: Interval-valued Atanassov intuitionistic fuzzy set; Interval linear order; Interval-valued Atanassov intuitionistic multi-expert decision making.

\section{Introduction}

In decision making problems it may happen that, after the exploitation phase, the best alternatives are equally ranked and it is not possible to decide which one is the best. It has been noticed [1] that these troubles often appear when the entries of the considered fuzzy preference matrix are close to 0.5 , that is, when the experts have doubts about their preferences of some alternatives over the others. In this situation, the systematic use of extensions of fuzzy sets has been shown to be a really useful tool [2]. Among those fuzzy sets, interval-valued fuzzy sets(IVFSs) [3-5] or, equivalently, Atanassov intuitionistic fuzzy sets(AIFs) [6] play indeed a crucial role.

In some special cases, despite the fact of using IVFSs and AIFs, still remain problems that are similar to those encountered in the previous ones. For these new last situations we may use the interval-valued Atanassov intuitionistic fuzzy sets (IVAIFSs) [7]. Besides, the use of intervals to represent membership and non-membership has, from our point of view, a double advantage:

1. If we want to model environments where there exist non-comparable elements, it will be enough to use classical partial orders between intervals. This is not the case in this work.

Email addresses: laura.demiguel@unavarra.es (L. De Miguel), bustince@unavarra.es (H. Bustince), fcojavier.fernandez@unavarra.es (J. Fernandez), steiner@unavarra.es (E. Induráin), anna.kolesarova@stuba.sk (A. Kolesárová), mesiar@math.sk (R. Mesiar) 
2. If we must represent ignorance [8] associated to the datum given by an expert, we can understand the length of the intervals as a representation of such ignorance. If, in these cases, we need to be able to compare any two data, then we can use any of the linear orders we consider here.

Once the decision of using IVAIFSs to deal with a decision making problem has been reached, we should choose, accordingly, a linear order between pairs of intervals. In this way, we will select as the best option the alternative which is associated to the largest pair of intervals, with respect to the considered linear order.

Moreover, in decision making problems we must also aggregate the information furnished by the experts by means of aggregation functions [9-11].

All these considerations have led us to aim the following objectives:

(1) To use aggregation functions for building linear orders for pairs of intervals whose end-points belong to the unit interval;

(2) To study methods for constructing linear orders on the set of IVAIFSs;

(3) To deal with the exploitation phase of decision making problems through IVAIFSs, by using the previously built linear orders.

The structure of this paper is the following. In Section 2 we introduce the notation and recall some well-known notions. In Sections 3-4, we construct two classes of linear orders between pairs of intervals. Section 5 contains an application of our theoretical results to group decision making. In particular, we provide two algorithms. Some concluding remarks as well as suggestions for further research close the paper.

\section{Previous concepts and results}

We start by recalling some well-known concepts that will be useful for subsequent developments throughout the paper.

\subsection{On orders and partially ordered sets}

Given a partially ordered set (poset) $(P, \preceq)$, we say that

a) $1_{P}$ is the top of the poset if for all $x \in P$ it holds $x \preceq 1_{P}$.

b) $0_{P}$ is the bottom of the poset if for all $x \in P$ it holds $0_{P} \preceq x$.

In case they exist, $1_{P}$ and $0_{P}$ are unique.

Let $K([0,1]) \subset \mathbb{R}^{2}$ be given by

$$
K([0,1])=\{(\underline{x}, \bar{x}) \in[0,1] \times[0,1] \mid \underline{x} \leq \bar{x}\}
$$

and let $L([0,1])$ be the set of all closed subintervals of the unit interval, that is

$$
L([0,1])=\{\mathbf{x} \mid \mathbf{x}=[\underline{x}, \bar{x}] \text { such that } 0 \leq \underline{x} \leq \bar{x} \leq 1\} .
$$

There is a straightforward bijection $i: K([0,1]) \longrightarrow L([0,1])$ given by $i((\underline{x}, \bar{x}))=[\underline{x}, \bar{x}]=\mathbf{x}$. Through this bijection, the partial order on $\mathbb{R}^{2},(a, b) \leq_{2}(c, d)$ if and only if $a \leq c$ and $b \leq d$ induces an equivalent partial order on $L([0,1])$, namely,

$$
\mathbf{x} \preceq_{2} \mathbf{y} \text { iff } \underline{x} \leq \underline{y} \text { and } \bar{x} \leq \bar{y} .
$$


In this way, $\left(L([0,1]), \preceq_{2}\right)$ is a poset whose bottom and top are, respectively, $\mathbf{0}=[0,0]$ and $\mathbf{1}=[1,1]$. In fact, the bijection above is a lattice isomorphism ${ }^{1}$.

We refer as $(L([0,1]))^{2}$, to the universe of pairs of intervals, that is,

$$
(L([0,1]))^{2}=\{(\mathbf{x}, \mathbf{y})=([\underline{x}, \bar{x}],[\underline{y}, \bar{y}]) \text { with } \underline{x}, \bar{x}, \underline{y}, \bar{y} \in[0,1]\} .
$$

Similarly to what happens in the case of $\mathbb{R}^{2}$ and $L([0,1])$, the partial order on $\mathbb{R}^{4}$, given by $\left(a_{1}, b_{1}, c_{1}, d_{1}\right) \leq_{4}\left(a_{2}, b_{2}, c_{2}, d_{2}\right)$ if and only if $a_{1} \leq a_{2}$ and $b_{1} \leq b_{2}$ and $c_{1} \leq c_{2}$ and $d_{1} \leq d_{2}$, also induces an equivalent partial order $\preceq_{4}$ on $(L([0,1]))^{2}$, given by

$$
\left(\mathbf{x}_{\mathbf{1}}, \mathbf{y}_{\mathbf{1}}\right) \preceq_{4}\left(\mathbf{x}_{\mathbf{2}}, \mathbf{y}_{\mathbf{2}}\right) \text { if and only if } \underline{x}_{1} \leq \underline{x}_{2} \text { and } \bar{x}_{1} \leq \bar{x}_{2} \text { and } \underline{y}_{1} \leq \underline{y}_{2} \text { and } \bar{y}_{1} \leq \bar{y}_{2} .
$$

In this way, $\left((L([0,1]))^{2}, \preceq_{4}\right)$ becomes a poset whose bottom and top are, respectively, $(\mathbf{0}, \mathbf{0})=$ $([0,0],[0,0])$ and $(\mathbf{1}, \mathbf{1})=([1,1],[1,1])$.

Definition 2.1. [18] An order $\preceq$ on $L([0,1])$ is said to be admissible if it is linear and refines the order $\preceq_{2}$, i.e., it is a linear order satisfying that for all $\boldsymbol{x}, \boldsymbol{y} \in L([0,1])$ such that $\boldsymbol{x} \preceq_{2} \boldsymbol{y}$ it holds $\boldsymbol{x} \preceq \boldsymbol{y}$.

Example 2.1. The lexicographic orders on $L([0,1])$, given by

- $\boldsymbol{x} \preceq_{\text {lex1 }} \boldsymbol{y}$ if and only if $(\underline{x}<\underline{y})$ or $(\underline{x}=\underline{y}$ and $\bar{x} \leq \bar{y})$ (lexicographic-1 order), and

- $\boldsymbol{x} \preceq_{\text {lex2 }} \boldsymbol{y}$ if and only if $(\bar{x}<\bar{y})$ or $(\bar{x}=\bar{y}$ and $\underline{x} \leq \underline{y})$ (lexicographic-2 order),

are admissible.

\subsection{Extensions of fuzzy sets}

Definition 2.2. [6] Let $U$ be a nonempty set usually called a universe. An Atanassov's Intuitionistic Fuzzy Set (AIFS) F over $U$ is given by

$$
F=\left\{\left\langle u, \mu_{F}(u), \nu_{F}(u)\right\rangle \mid u \in U\right\}
$$

where $\mu_{F}: U \rightarrow[0,1]$ defines the membership degree of the element $u \in U$ to $F$ and $\nu_{F}: U \rightarrow[0,1]$ defines its nonmembership degree to the same set $F$. Besides, the functions $\mu_{F}$ and $\nu_{F}$ satisfy that, for all $u \in U, \mu_{F}(u)+\nu_{F}(u) \leq 1$.

The pair $\left(\mu_{F}(u), \nu_{F}(u)\right)$ is called an intuitionistic pair, $\mathcal{L}([0,1])$ being the set of all possible intuitionistic pairs, i.e.,

$$
\mathcal{L}([0,1])=\left\{\mathbf{a} \mid \mathbf{a}=\left(a_{1}, a_{2}\right), a_{1}, a_{2} \in[0,1] \text { and } a_{1}+a_{2} \leq 1\right\} .
$$

In [6], Atanassov introduced a partial order in the universe of AIFSs.

\footnotetext{
${ }^{1}$ This kind of sets, namely $K([0,1])$ and $L([0,1])$ have already been used, suitably equipped with some order and latticial structure $[12,13]$, to construct some universal codomain where it was possible to represent different kinds of orderings as, e.g., total preorders, interval-orders and semiorders by means of a single function that preserves the ordinal structure. The bijection $i: K([0,1]) \longrightarrow L([0,1])$ has also been considered in those approaches, and some other similar bijections and/or latticial isomorphims as well as order isotonies have also been introduced accordingly. By the way, another universal codomain to represent different kinds of orderings, which is essentially equivalent to $K([0,1])$, consists of triangular and symmetric fuzzy numbers. For further information see [14-17].
} 
Definition 2.3. Let $F_{1}, F_{2}$ be two AIFSs. According to the order given by Atanassov in [6]

$$
F_{1} \leq F_{2} \text { if and only if for all } u \in U, \mu_{F_{1}}(u) \leq \mu_{F_{2}}(u) \text { and } \nu_{F_{1}}(u) \geq \nu_{F_{2}}(u) .
$$

Definition 2.4. [7] Let $U$ be a universe. An Interval-Valued Atanassov Intuitionistic Fuzzy Set (IVAIFS) $G$ over $U$ is given by

$$
G=\left\{\left\langle u, \mathrm{~m}_{\mathrm{G}}(u), \mathrm{n}_{\mathrm{G}}(u)\right\rangle \mid u \in U\right\}
$$

where $\mathrm{m}_{\mathrm{G}}: U \rightarrow L([0,1])$ defines the membership degree of the element $u \in U$ to $F$ and $\mathrm{n}_{\mathrm{G}}: U \rightarrow$ $L([0,1])$ defines its nonmembership degree to the same universe $U$. Moreover, for all $u \in U$, the sum of the upper boundary values of $\mathrm{m}_{\mathrm{G}}(u)$ and $\mathrm{n}_{\mathrm{G}}(u)$ must be lower than or equal to 1 .

The pair $\left(\mathrm{m}_{\mathrm{G}}(u), \mathrm{n}_{\mathrm{G}}(u)\right)$ is called an interval-valued intuitionistic pair, being $\mathcal{L}_{I V}([0,1])$ the set of all possible interval-valued intuitionistic pairs, i.e.,

$$
\mathcal{L}_{I V}([0,1])=\{(\mathbf{x}, \mathbf{y}), \text { with } \mathbf{x}, \mathbf{y} \in L([0,1]) \text { and } \bar{x}+\bar{y} \leq 1\} .
$$

Remark 1. Note that $\mathcal{L}_{I V}([0,1])$ consists of special types of intervals, while $(\mathcal{L}([0,1]))^{2}$ is a set of all possible intuitionistic pairs.

Definition 2.5. Let $G_{1}, G_{2}$ be two IVAIFSs. According to the order given by Atanassov in [7], $G_{1} \preceq G_{2}$ if and only if, for all $u \in U$,

$$
\mathrm{m}_{\mathrm{G} 1}(u) \preceq_{2} \mathrm{~m}_{\mathrm{G} 2}(u) \text { and } \mathrm{n}_{\mathrm{G} 2}(u) \preceq_{2} \mathrm{n}_{\mathrm{G} 1}(u),
$$

where $\preceq_{2}$ is the partial order on $L([0,1])$ given in Equation (1).

\subsection{Aggregation functions}

Definition 2.6. Given a poset $\left(P, \preceq_{P}\right)$ with bottom $0_{P}$ and top $1_{P}$, an aggregation function $M$ on $P$ w.r.t the order $\preceq_{P}$ (also known as an $\preceq_{P}$-aggregation function) is a mapping $M: P^{n} \rightarrow P$ satisfying

- $M\left(0_{P}, \ldots, 0_{P}\right)=0_{P}, \quad M\left(1_{P}, \ldots, 1_{P}\right)=1_{P}$, and

- $M\left(x_{1}, \ldots, x_{n}\right) \preceq_{P} M\left(y_{1}, \ldots, y_{n}\right)$ for $\left(x_{1}, \ldots, x_{n}\right) \preceq_{P}\left(y_{1}, \ldots, y_{n}\right)$

where $\left(x_{1}, \ldots, x_{n}\right) \preceq_{P}\left(y_{1}, \ldots, y_{n}\right)$ holds if and only if $x_{i} \preceq_{P} y_{i}$ for all $i \in\{1, \ldots, n\}$.

This definition extends the usual one for the unit interval [0,1]. For further information see [19].

Proposition 2.1. [18] Let $B_{1}, B_{2}:[0,1]^{2} \rightarrow[0,1]$ be two continuous aggregation functions, such that for all $\left(p_{1}, p_{2}\right),\left(q_{1}, q_{2}\right) \in K([0,1])$, the equalities $B_{1}\left(p_{1}, p_{2}\right)=B_{1}\left(q_{1}, q_{2}\right)$ and $B_{2}\left(p_{1}, p_{2}\right)=$ $B_{2}\left(q_{1}, q_{2}\right)$ only hold provided that $\left(p_{1}, p_{2}\right)=\left(q_{1}, q_{2}\right)$.

The order $\preceq_{B_{1}, B_{2}}$ on $L([0,1])$, given by

$\boldsymbol{x} \preceq_{B_{1}, B_{2}} \boldsymbol{y}$ if and only if $B_{1}(\underline{x}, \bar{x})<B_{1}(\underline{y}, \bar{y})$ or else $\left(B_{1}(\underline{x}, \bar{x})=B_{1}(\underline{y}, \bar{y})\right.$ and $\left.B_{2}(\underline{x}, \bar{x}) \leq B_{2}(\underline{y}, \bar{y})\right)$, is an admissible order on $L([0,1])$.

The following results can be found in $[9,11,20,21]$. 
Definition 2.7. A function $T:[0,1]^{2} \rightarrow[0,1]$ is called a $t$-norm if it is symmetric, associative, increasing with respect to the order $\leq$ and $T(x, 1)=x$ for all $x \in[0,1]$.

Definition 2.8. A function $S:[0,1]^{2} \rightarrow[0,1]$ is called a $t$-conorm if it is symmetric, associative, increasing with respect to the order $\leq$ and $S(x, 0)=x$ for all $x \in[0,1]$.

A strictly decreasing and continuous function $n:[0,1] \rightarrow[0,1]$ such that $n(0)=1$ and $n(1)=0$ is called a strict negation. If, in addition, it is involutive (that is, $n(n(x))=x$ for all $x \in[0,1]$ ), then $n$ is said to be a strong negation. A t-norm $T$ is dual to a t-conorm $S$ (and vice-versa) with respect to a strong negation $n$ if $T(x, y)=n(S(n(x), n(y)))$ for all $x, y \in[0,1]$.

\section{Admissible orders on $(L([0,1]))^{2}$}

Although a partial order is enough to define aggregation functions, some special classes of aggregations actually require to have at hand a linear order. Examples of such classes are Choquet integrals and Sugeno integrals. The order given by Atanassov for IVAIFSs is partial, which is a undeniable handicap in the adaptation of such classes of aggregation operators to the IVAI setup. In this section we define the admissible linear orders on $(L([0,1]))^{2}$, generalizing the concept of admissible orders on $L([0,1])$.

Definition 3.1. An order $\preceq$ on $(L([0,1]))^{2}$ is said to be admissible if it is a linear and refines the order $\preceq_{4}$ in Eq. (2), i.e., it is linear order satisfying that for all $\left(\boldsymbol{x}_{1}, \boldsymbol{y}_{1}\right),\left(\boldsymbol{x}_{2}, \boldsymbol{y}_{2}\right) \in(L([0,1]))^{2}$, $\left(\boldsymbol{x}_{1}, \boldsymbol{y}_{1}\right) \preceq_{4}\left(\boldsymbol{x}_{2}, \boldsymbol{y}_{2}\right)$ implies $\left(\boldsymbol{x}_{1}, \boldsymbol{y}_{1}\right) \preceq\left(\boldsymbol{x}_{2}, \boldsymbol{y}_{2}\right)$.

The elements $z_{i}=\left(\mathbf{x}_{\mathbf{i}}, \mathbf{y}_{\mathbf{i}}\right) \in(L([0,1]))^{2}$ can be visualized in a straightforward manner. Since $\mathbf{x}_{\mathbf{i}}, \mathbf{y}_{\mathbf{i}} \in L([0,1])$, each pair of intervals can be drawn as a rectangle for which the first interval lies in the horizontal axis and the second interval lies in the vertical one. In such a representation, the following statements hold true:

- The wider the first interval, the wider the rectangle.

- The wider the second interval, the higher the rectangle.

As a consequence, the area of the rectangle will be directly proportional to the width of the intervals. Furthermore, for any $z_{1}, z_{2} \in(L([0,1]))^{2}, z_{1} \preceq_{4} z_{2}$ if and only if each corner of the rectangle of $z_{2}$ is located above and on the right side of its corresponding corner in the rectangle $z_{1}$.

Example 3.1. Let $z_{1}=([0.3,0.6],[0.2,0.7]), z_{2}=([0.5,0.8],[0.55,0.9]), z_{3}=([0.4,0.5],[0.3,0.35])$, $z_{4}=([0.1,0.4],[0.4,0.6])$. The intervals can be represented in the unit square $[0,1]^{2}$ as in Fig. 1 . In that figure some visual interpretations can be drawn. For example, we have that the intervals of $z_{1}$ are wider than those of any other $z_{i}$, since its area is significantly greater. Alternatively, we have that $z_{i} \preceq_{4} z_{2}$ for $i \in\{1,3,4\}$, since the corners of $z_{2}$ are located above and on the right side w.r.t the other rectangles. Similarly, we can deduce that $z_{1}, z_{3}$ and $z_{4}$ are incomparable in terms of $\preceq_{4}$.

In [18], Bustince et al. introduced a construction method of admissible orders on $L([0,1])$ by using two aggregation functions. Such method can also be generalized to handle elements in $(L([0,1]))^{2}$. 


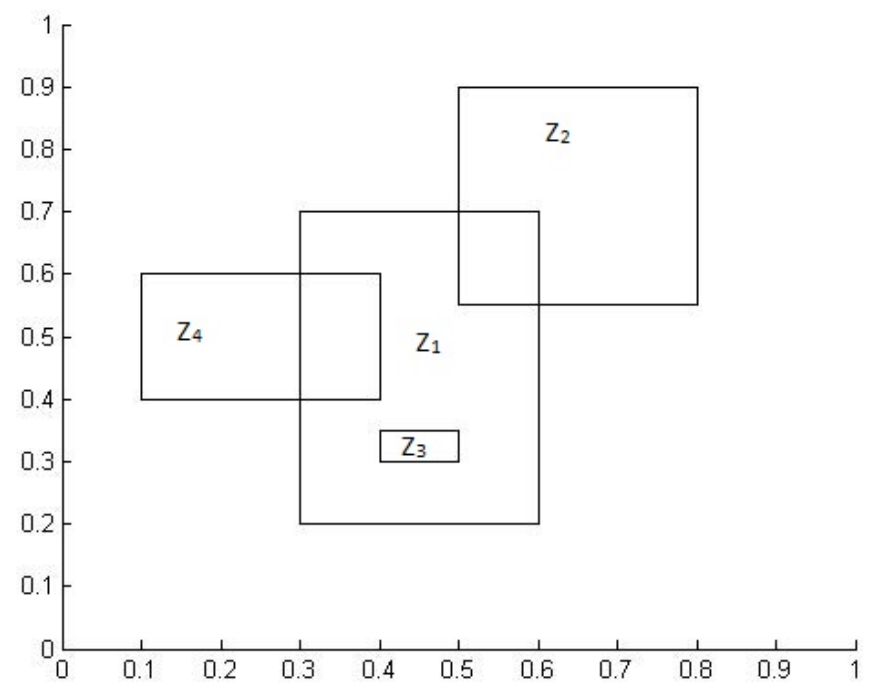

Figure 1: Pairs of intervals

Proposition 3.1. Let $A=<A_{1}, A_{2}, A_{3}, A_{4}>$ be four aggregation functions ${ }^{2}, A_{i}:[0,1]^{4} \rightarrow[0,1]$ such that for all $(\boldsymbol{p}, \boldsymbol{q}),(\boldsymbol{r}, \boldsymbol{s}) \in(L([0,1]))^{2}$ the equalities $A_{i}(p, \bar{p}, q, \bar{q})=A_{i}(\underline{r}, \bar{r}, \underline{s}, \bar{s})$ for all $i=$ $\{1, \ldots 4\}$ only hold if $(\boldsymbol{p}, \boldsymbol{q})=(\boldsymbol{r}, \boldsymbol{s})$.

An admissible order $\preceq_{A}$ on $(L([0,1]))^{2}$ can be defined as follows

$\left(\boldsymbol{x}_{1}, \boldsymbol{y}_{1}\right) \preceq_{A}\left(\boldsymbol{x}_{2}, \boldsymbol{y}_{2}\right)$ if and only if one of the (mutually exclusive) following conditions is satisfied.

i) $A_{1}\left(\underline{x}_{1}, \bar{x}_{1}, \underline{y}_{1}, \bar{y}_{1}\right)<A_{1}\left(\underline{x}_{2}, \bar{x}_{2}, \underline{y}_{2}, \bar{y}_{2}\right)$;

ii) $A_{1}\left(\underline{x}_{1}, \bar{x}_{1}, \underline{y}_{1}, \bar{y}_{1}\right)=A_{1}\left(\underline{x}_{2}, \bar{x}_{2}, \underline{y}_{2}, \bar{y}_{2}\right)$ and $A_{2}\left(\underline{x}_{1}, \bar{x}_{1}, \underline{y}_{1}, \bar{y}_{1}\right)<A_{2}\left(\underline{x}_{2}, \bar{x}_{2}, \underline{y}_{2}, \bar{y}_{2}\right)$;

iii) $A_{1}\left(\underline{x}_{1}, \bar{x}_{1}, \underline{y}_{1}, \bar{y}_{1}\right)=A_{1}\left(\underline{x}_{2}, \bar{x}_{2}, \underline{y}_{2}, \bar{y}_{2}\right)$ and $A_{2}\left(\underline{x}_{1}, \bar{x}_{1}, \underline{y}_{1}, \bar{y}_{1}\right)=A_{2}\left(\underline{x}_{2}, \bar{x}_{2}, \underline{y}_{2}, \bar{y}_{2}\right)$ and $A_{3}\left(\underline{x}_{1}, \bar{x}_{1}, \underline{y}_{1}, \bar{y}_{1}\right)<A_{3}\left(\underline{x}_{2}, \bar{x}_{2}, \underline{y}_{2}, \bar{y}_{2}\right)$;

iv) $A_{1}\left(\underline{x}_{1}, \bar{x}_{1}, \underline{y}_{1}, \bar{y}_{1}\right)=A_{1}\left(\underline{x}_{2}, \bar{x}_{2}, \underline{y}_{2}, \bar{y}_{2}\right)$ and $A_{2}\left(\underline{x}_{1}, \bar{x}_{1}, \underline{y}_{1}, \bar{y}_{1}\right)=A_{2}\left(\underline{x}_{2}, \bar{x}_{2}, \underline{y}_{2}, \bar{y}_{2}\right)$ and $A_{3}\left(\underline{x}_{1}, \bar{x}_{1}, \underline{y}_{1}, \bar{y}_{1}\right)=A_{3}\left(\underline{x}_{2}, \bar{x}_{2}, \underline{y}_{2}, \bar{y}_{2}\right)$ and $A_{4}\left(\underline{x}_{1}, \bar{x}_{1}, \underline{y}_{1}, \bar{y}_{1}\right) \leq A_{4}\left(\underline{x}_{2}, \bar{x}_{2}, \underline{y}_{2}, \bar{y}_{2}\right)$.

Proof. The order $\preceq_{A}$ refines $\leq_{4}$ since every $A_{i}$ is an aggregation function. Moreover, the linearity is assured since the four equalities of $A_{i}$ only hold simultaneously if $\left(\mathbf{x}_{1}, \mathbf{y}_{\mathbf{1}}\right)=\left(\mathbf{x}_{\mathbf{2}}, \mathbf{y}_{\mathbf{2}}\right)$. The transitivity follows from the transitivity of the standard order on $[0,1]$.

Remark 2. Notice that any permutation of the aggregation functions $A_{i}$ also produces an admissible order different from the former one.

${ }^{2}$ Warning: notice that here the order of appearence of the $A_{i}^{\prime} s$ counts. See also Remark 2 
Remark 3. In [18] it was proven that an admissible order on $K([0,1])$ can not be induced by a single function. Clearly, this result also holds true since we are working in a larger space.

Henceforward, we use the order generated by four aggregation functions (in Prop 3.1). Thus, all the ideas to be introduced till the end of this section refer to such family of admissible orders named 4-admissible.

Example 3.2. The lexicographic orders can be constructed from the four projections.

1. The standard lexicographic order: let $A_{i}$ be the aggregation function that maps to the $i$-th component (i.e. the $i$-th projection). In that case, $\left(\boldsymbol{x}_{1}, \boldsymbol{y}_{1}\right) \preceq_{A}\left(\boldsymbol{x}_{2}, \boldsymbol{y}_{2}\right)$ if and only if

- $\left(\underline{x}_{1}<\underline{x}_{2}\right)$, or

- $\left(\underline{x}_{1}=\underline{x}_{2}\right.$ and $\left.\bar{x}_{1}<\bar{x}_{2}\right)$, or

- $\left(\underline{x}_{1}=\underline{x}_{2}, \bar{x}_{1}=\bar{x}_{2}\right.$ and $\left.\underline{y}_{1}<\bar{y}_{2}\right)$, or

- $\left(\underline{x}_{1}=\underline{x}_{2}, \bar{x}_{1}=\bar{x}_{2}, \underline{y}_{1}=\underline{y}_{2}\right.$ and $\left.\bar{y}_{1} \leq \bar{y}_{2}\right)$.

2. The reversed lexicographic order: let $A_{i}$ be the aggregation function that maps to the $(5-i)$-th component (i.e. the $(5-i)$-th projection). In that case, $\left(\boldsymbol{x}_{1}, \boldsymbol{y}_{1}\right) \preceq_{A}\left(\boldsymbol{x}_{2}, \boldsymbol{y}_{2}\right)$ if and only if

- $\left(\bar{y}_{1}<\bar{y}_{2}\right)$, or

- $\left(\bar{y}_{1}=\bar{y}_{2}\right.$ and $\left.\underline{y}_{1}<\underline{y}_{2}\right)$, or

- $\left(\bar{y}_{1}=\bar{y}_{2}, \underline{y}_{1}=\underline{y}_{2}\right.$ and $\left.\bar{x}_{1}<\bar{x}_{2}\right)$, or

- $\left(\bar{y}_{1}=\bar{y}_{2}, \underline{y}_{1}=\underline{y}_{2}, \bar{x}_{1}=\bar{x}_{2}\right.$ and $\left.\underline{x}_{1} \leq \underline{x}_{2}\right)$.

3. Any other permutation of the projections gives rise to an admissible order where we compare the components in a predetermined order.

Proposition 3.2. Let $A=<A_{1}, A_{2}, A_{3}, A_{4}>$ be four aggregation functions given by

$$
A_{i}\left(\underline{x}_{1}, \bar{x}_{1}, \underline{y}_{1}, \bar{y}_{1}\right)=a_{i} \underline{x}_{1}+b_{i} \bar{x}_{1}+c_{i} \underline{y}_{1}+d_{i} \bar{y}_{1},
$$

with $a_{i}, b_{i}, c_{i}, d_{i} \in[0,1], a_{i}+b_{i}+c_{i}+d_{i}=1$ and

$$
|D|=\left|\begin{array}{llll}
a_{1} & b_{1} & c_{1} & d_{1} \\
a_{2} & b_{2} & c_{2} & d_{2} \\
a_{3} & b_{3} & c_{3} & d_{3} \\
a_{4} & b_{4} & c_{4} & d_{4}
\end{array}\right| \neq 0 .
$$

Then (and only then), the order generated by the aggregation functions $A_{i}$ is a 4-admissible order.

Proof.

The functions $A_{i}$ are weighted arithmetic means. Let $\left(\left[\underline{x}_{1}, \bar{x}_{1}\right],\left[\underline{y}_{1}, \bar{y}_{1}\right]\right),\left(\left[\underline{x}_{2}, \bar{x}_{2}\right],\left[\underline{y}_{2}, \bar{y}_{2}\right]\right) \in$ $(L([0,1]))^{2}$, such that

$$
a_{i} \underline{x}_{1}+b_{i} \bar{x}_{1}+c_{i} \underline{y}_{1}+d_{i} \bar{y}_{1}=a_{i} \underline{x}_{2}+b_{i} \bar{x}_{2}+c_{i} \underline{y}_{2}+d_{i} \bar{y}_{2}
$$

for $i \in\{1, \ldots, 4\}$. Because of the regularity of $D$, both linear systems have a unique and common solution, i.e., $\left(\underline{x}_{1}, \bar{x}_{1}, \underline{y}_{1}, \bar{y}_{1}\right)=\left(\underline{x}_{2}, \bar{x}_{2}, \underline{y}_{2}, \bar{y}_{2}\right)$. The result now follows from Prop. 3.1. 
Example 3.3. Let A contain the following aggregation functions:

- $A_{1}\left(\underline{x}_{1}, \bar{x}_{1}, \underline{y}_{1}, \bar{y}_{1}\right)=\frac{3}{8} \underline{x}_{1}+\frac{3}{8} \bar{x}_{1}+\frac{1}{8} \underline{y}_{1}+\frac{1}{8} \bar{y}_{1}$;

- $A_{2}\left(\underline{x}_{1}, \bar{x}_{1}, \underline{y}_{1}, \bar{y}_{1}\right)=\frac{10}{20} \underline{x}_{1}+\frac{5}{20} \bar{x}_{1}+\frac{3}{20} \underline{y}_{1}+\frac{2}{20} \bar{y}_{1}$;

- $A_{3}\left(\underline{x}_{1}, \bar{x}_{1}, \underline{y}_{1}, \bar{y}_{1}\right)=\frac{1}{20} \underline{x}_{1}+\frac{10}{20} \bar{x}_{1}+\frac{8}{20} \underline{y}_{1}+\frac{1}{20} \bar{y}_{1}$;

- $A_{4}\left(\underline{x}_{1}, \bar{x}_{1}, \underline{y}_{1}, \bar{y}_{1}\right)=\frac{1}{4} \underline{x}_{1}+\frac{1}{4} \bar{x}_{1}+\frac{1}{4} \underline{y}_{1}+\frac{1}{4} \bar{y}_{1}$.

Since $|D|=-0.0069$, the order generated by $A$, as in Prop. 3.1, is a 4-admissible order.

Remark 4. Notice that the value of the determinant is close to 0 but this is due to the fact that all the elements of the matrix are smaller than 1.

The construction of admissible orders through a 4-tuple of weighted arithmetic means has an interesting geometrical interpretation. If we consider $A$ in the form of the corresponding four weighting vectors which generate $A_{1}, \ldots, A_{4}$, i.e.,

$$
A \approx R=\left\{<a_{1}, b_{1}, c_{1}, d_{1}>,<a_{2}, b_{2}, c_{2}, d_{2}>,<a_{3}, b_{3}, c_{3}, d_{3}>,<a_{4}, b_{4}, c_{4}, d_{4}>\right\}
$$

the condition in Prop. 3.2 means that $R$ is a basis of the vector space $\mathbb{R}^{4}$. Hence, to any basis $R$ of $\mathbb{R}^{4}$ which consists of weighting vectors there is a unique admissible order $\preceq_{A}$ constructed by means of the corresponding weighted means.

Finally, after changing the basis, the values of interval-valued intuitionistic pairs in the new basis, (which are now in $[0,1]^{4}$ ), are ordered through the standard lexicographic order.

Proposition 3.3. Let a tuple $A=\left\langle A_{1}, \ldots, A_{4}\right\rangle$ of aggregation functions generate an admissible order $\preceq_{A}$. Let $B_{i}:[0,1]^{2} \longrightarrow[0,1], i \in\{1, \ldots, 4\}$ be four aggregation functions such that

- $A_{i}(\underline{x}, \bar{x}, \underline{y}, \bar{y})=B_{i}(\underline{x}, \bar{x})$ for $i \in\{1,2\}$, and

- $A_{j}(\underline{x}, \bar{x}, \underline{y}, \bar{y})=B_{j}(\underline{y}, \bar{y})$ for $j \in\{3,4\}$.

Then, $\left(\boldsymbol{x}_{1}, \boldsymbol{y}_{1}\right) \preceq_{A}\left(\boldsymbol{x}_{2}, \boldsymbol{y}_{2}\right)$ if and only if

i) $\left(\boldsymbol{x}_{1} \prec_{B_{1}, B_{2}} \boldsymbol{x}_{2}\right)$, or

ii) ( $\boldsymbol{x}_{\boldsymbol{1}}=\boldsymbol{x}_{2}$ and $\left.\boldsymbol{y}_{1} \preceq_{B_{3}, B_{4}} \boldsymbol{y}_{2}\right)$,

where $\preceq_{B_{i}, B_{j}}$ is the order on $L([0,1])$ generated in Prop. 2.1.

Proof. It is straightforward.

Notice that, if we use $B_{1}=B_{3}$ and $B_{2}=B_{4}$, the result is a 4-admissible order where we combine the standard lexicographic order with the order $\preceq_{B_{1}, B_{2}}$. The resulting order acts as follows: first we compare the intervals using $\preceq_{B_{1}, B_{2}}$ and, only if they are equal, we compare the second interval with that same order $\left(\preceq_{B_{1}, B_{2}}\right)$. For instance, the standard lexicographic order can be seen as the composition of the lexicographic-1 order between intervals combined with itself. 
Alternatively, notice that, if $A_{i}(\underline{x}, \bar{x}, \underline{y}, \bar{y})=B_{i}(\underline{y}, \bar{y})$ for $i \in\{1,2\}$, and $A_{j}(\underline{x}, \bar{x}, \underline{y}, \bar{y})=B_{j}(\underline{x}, \bar{x})$ for $j \in\{3,4\}$, then the resulting order is also 4 -admissible.

A well-known class of binary aggregation functions is that of Atanassov's operators $\mathbb{K}_{\alpha}$ given by $\mathbb{K}_{\alpha}(a, b)=a+\alpha(b-a)$ with $\alpha \in[0,1]$.

In our particular case, the inputs being intervals, an Atanassov's operator acting on the endpoints of the intervals yields a point inside the corresponding intervals.

Example 3.4. Let $\alpha_{1}, \alpha_{2}, \alpha_{3}, \alpha_{4} \in[0,1]$, with $\alpha_{1} \neq \alpha_{2}$ and $\alpha_{3} \neq \alpha_{4}$. Let $A=<A_{1}, \ldots, A_{4}>$ be four aggregation functions given by

- $A_{i}\left(\underline{x}_{1}, \bar{x}_{1}, \underline{y}_{1}, \bar{y}_{1}\right)=\mathbb{K}_{\alpha_{i}}\left(\underline{x}_{1}, \bar{x}_{1}\right)$, for $i \in\{1,2\}$, and

- $A_{j}\left(\underline{x}_{1}, \bar{x}_{1}, \underline{y}_{1}, \bar{y}_{1}\right)=\mathbb{K}_{\alpha_{j}}\left(\underline{y}_{1}, \bar{y}_{1}\right)$, for $j \in\{3,4\}$.

The tuple A generates a 4-admissible order that renders in $\left(\boldsymbol{x}_{\boldsymbol{1}}, \boldsymbol{y}_{\mathbf{1}}\right) \preceq_{A}\left(\boldsymbol{x}_{2}, \boldsymbol{y}_{2}\right)$ if and only if

- $\left(\boldsymbol{x}_{1} \prec_{\mathbb{K}_{\alpha_{1}}, \mathbb{K}_{\alpha_{2}}} \boldsymbol{x}_{2}\right)$, or

- $\left(\boldsymbol{x}_{1}=\mathbb{K}_{\alpha_{1}}, \mathbb{K}_{\alpha_{2}} \boldsymbol{x}_{2}\right.$ and $\left.\boldsymbol{y}_{1} \preceq_{\mathbb{K}_{\alpha_{3}}, \mathbb{K}_{\alpha_{4}}} \boldsymbol{y}_{2}\right)$.

From the construction in Example 3.4, we can retrieve some well-known orders. For example, if $\left\{\alpha_{1}, \alpha_{2}\right\}=\{0,1\}$ and $\left\{\alpha_{3}, \alpha_{4}\right\}=\{0,1\}$, we obtain lexicographic orders. Moreover, all these 4-admissible orders are particular examples of the construction in Prop. 3.2, with $c=d=0$ for $A_{1}$, $A_{2}$ and $a=b=0$ for $A_{3}$ and $A_{4}$.

In [18] it was proven that given an $\alpha \in[0,1)$ then all admissible orders $\preceq_{\alpha, \beta}$ on $L([0,1])$ with $\beta>\alpha$ coincide. Then, different aggregation functions could generate the same admissible order. This also affects to admissible orders generated as in Prop. 3.2. For instance,

$$
\left|D_{1}\right|=\left|\begin{array}{cccc}
\frac{1}{2} & \frac{1}{2} & 0 & 0 \\
0 & 1 & 0 & 0 \\
0 & 0 & \frac{1}{2} & \frac{1}{2} \\
0 & 0 & 0 & 1
\end{array}\right| \neq 0, \quad\left|D_{2}\right|=\left|\begin{array}{cccc}
\frac{1}{2} & \frac{1}{2} & 0 & 0 \\
\frac{1}{3} & \frac{1}{3} & 0 & 0 \\
0 & 0 & \frac{1}{2} & \frac{1}{2} \\
0 & 0 & \frac{1}{3} & \frac{2}{3}
\end{array}\right| \neq 0
$$

generate the same order.

\section{IVAIF-admissible order on $\mathcal{L}_{I V}([0,1])$}

The admissible orders defined in Section 3 refine the partial order $\preceq_{4}$. However, any of them could also refine the partial order given by Atanassov for IVAIFS [7]. In this section, we define a new family of linear orders with a crucial additional feature, namely, they refine Atanassov's partial order.

We remind the reader that in Atanassov's partial order, given two elements $\left(\mathbf{x}_{\mathbf{1}}, \mathbf{y}_{\mathbf{1}}\right),\left(\mathbf{x}_{\mathbf{2}}, \mathbf{y}_{\mathbf{2}}\right) \in$ $\mathcal{L}_{I V}([0,1])$

$$
\left(\mathbf{x}_{\mathbf{1}}, \mathbf{y}_{\mathbf{1}}\right) \preceq\left(\mathbf{x}_{\mathbf{2}}, \mathbf{y}_{\mathbf{2}}\right) \text { if and only if } \underline{x}_{1} \leq \underline{x}_{2}, \bar{x}_{1} \leq \bar{x}_{2}, \underline{y}_{1} \geq \underline{y}_{2} \text {, and } \bar{y}_{1} \geq \bar{y}_{2} .
$$

Definition 4.1. An order $\preceq$ on $\mathcal{L}_{I V}([0,1])$ is said to be an IVAIF-admissible order if it is a linear order and refines the partial order given by Atanassov for IVAIFS (Eq.(3)). 


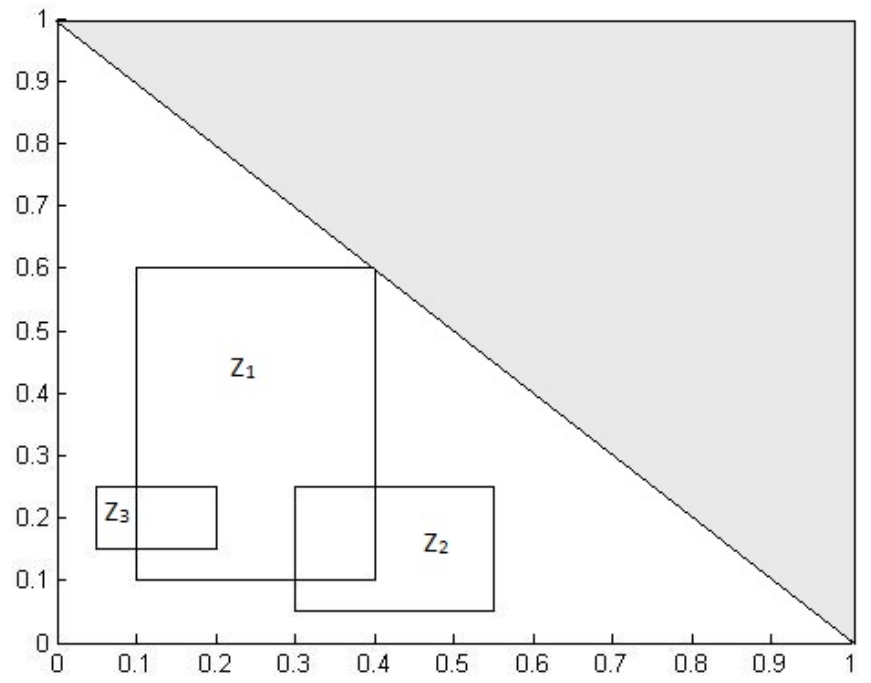

Figure 2: Visual representation of interval-valued intuitionistic pairs. The white zone represents the subset of $\mathcal{L}_{I V}([0,1])$ in which such pairs are allowed.

Notice that, if we have an IVAIF-admissible order on $\mathcal{L}_{I V}([0,1])$, as in Def. 4.1, then the bottom of $\left(\mathcal{L}_{I V}([0,1]), \preceq\right)$ is $(\mathbf{0}, \mathbf{1})$ and the top is $(\mathbf{1}, \mathbf{0})$.

As in Section 3, we can generate a visualization of the elements in $\mathcal{L}_{I V}([0,1]) \subset(L([0,1]))^{2}$ that captures the behaviour of the admissible orders in Def. 4.1. Following the visualization rules in Fig. 1 we have that, for any two elements $z_{1}, z_{2}$ in $\mathcal{L}_{I V}([0,1]), z_{1} \preceq z_{2}$ if and only if the corners of $z_{2}$ are individually located below and to the right of those of $z_{1}$. For example, in Fig. 2, we have given $z_{1}=([0.1,0.4],[0.1,0.6]), z_{2}=([0.3,0.55],[0.05,0.25]), z_{3}=([0.05,0.2],[0.15,0.25]) \in \mathcal{L}_{I V}([0,1])$. Visually, it is evident that $z_{1} \preceq z_{2}$ and $z_{3} \preceq z_{2}$, but also that $z_{1}$ and $z_{3}$ are not comparable with the partial order in Def. 4.1. Notice that, in this visualization, no element is allowed to be in the grey zone of the rectangle in Fig. 2 due to the restrictions in the definitions of the membership and nonmembership degrees in an interval-valued intuitionistic pair.

In the sequel, two different constructions of IVAIF-admissible orders are introduced.

Proposition 4.1. Let $B=<B_{1}, B_{2}, B_{3}, B_{4}>$ be four aggregation functions $B_{i}:[0,1]^{4} \longrightarrow[0,1]$ which generate the orders $\preceq_{B_{1}, B_{2}}$ and $\preceq_{B_{3}, B_{4}}$ on $L([0,1])$ as in Prop. 2.1. Then the order relation $\preceq_{B}^{I V}$ given by

$$
\left(\boldsymbol{x}_{1}, \boldsymbol{y}_{1}\right) \preceq_{B}^{I V}\left(\boldsymbol{x}_{2}, \boldsymbol{y}_{2}\right) \text { if and only if } \boldsymbol{x}_{1} \prec_{B_{1}, B_{2}} \boldsymbol{x}_{2} \text { or }\left(\boldsymbol{x}_{1}=\boldsymbol{x}_{2} \text { and } \boldsymbol{y}_{2} \preceq_{B_{3}, B_{4}} \boldsymbol{y}_{1}\right)
$$

is an IVAIF-admissible order.

Proof. The linearity of $\preceq_{B}^{I V}$ is straight as $\mathcal{L}_{I V}([0,1]) \subset(L([0,1]))^{2}$. In addition, it refines the partial order given by Atanassov due to the fact that the order relation, $\preceq_{B_{3}, B_{4}}$, has been reversed.

In particular, if $B_{1}=B_{3}$ and $B_{2}=B_{4}$, then $\preceq_{B_{1}, B_{2}}=\preceq_{B_{3}, B_{4}}$ and, consequently, the same order is used to compare both intervals although in the second one the order is reversed. 
Proposition 4.2. Let $A=<A_{1}, A_{2}, A_{3}, A_{4}>$ be four aggregation functions, $A_{i}:[0,1]^{4} \rightarrow[0,1]$ such that for all $\left(p_{1}, p_{2}, p_{3}, p_{4}\right),\left(q_{1}, q_{2}, q_{3}, q_{4}\right) \in[0,1]^{4}$ the equalities $A_{i}\left(p_{1}, p_{2}, p_{3}, p_{4}\right)=A_{i}\left(q_{1}, q_{2}, q_{3}, q_{4}\right)$ for all $i \in\{1, \ldots, 4\}$ only hold if $\left(p_{1}, p_{2}, p_{3}, p_{4}\right)=\left(q_{1}, q_{2}, q_{3}, q_{4}\right)$.

An IVAIF-admissible order $\preceq_{A}^{I V}$ on $\mathcal{L}_{I V}([0,1])$, is defined as follows: $\left(\boldsymbol{x}_{1}, \boldsymbol{y}_{1}\right) \preceq_{A}^{I V}\left(\boldsymbol{x}_{2}, \boldsymbol{y}_{2}\right)$ if and only if one of the following (mutually exclusive) conditions is satisfied.

i) $A_{1}\left(\underline{x}_{1}, \bar{x}_{1}, 1-\underline{y}_{1}, 1-\bar{y}_{1}\right)<A_{1}\left(\underline{x}_{2}, \bar{x}_{2}, 1-\underline{y}_{2}, 1-\bar{y}_{2}\right)$,

ii) $A_{1}\left(\underline{x}_{1}, \bar{x}_{1}, 1-\underline{y}_{1}, 1-\bar{y}_{1}\right)=A_{1}\left(\underline{x}_{2}, \bar{x}_{2}, 1-\underline{y}_{2}, 1-\bar{y}_{2}\right)$ and $A_{2}\left(\underline{x}_{1}, \bar{x}_{1}, 1-\underline{y}_{1}, 1-\bar{y}_{1}\right)<A_{2}\left(\underline{x}_{2}, \bar{x}_{2}, 1-\underline{y}_{2}, 1-\bar{y}_{2}\right)$,

iii) $A_{1}\left(\underline{x}_{1}, \bar{x}_{1}, 1-\underline{y}_{1}, 1-\bar{y}_{1}\right)=A_{1}\left(\underline{x}_{2}, \bar{x}_{2}, 1-\underline{y}_{2}, 1-\bar{y}_{2}\right)$, $A_{2}\left(\underline{x}_{1}, \bar{x}_{1}, 1-\underline{y}_{1}, 1-\bar{y}_{1}\right)=A_{2}\left(\underline{x}_{2}, \bar{x}_{2}, 1-\underline{y}_{2}, 1-\bar{y}_{2}\right)$, and $A_{3}\left(\underline{x}_{1}, \bar{x}_{1}, 1-\underline{y}_{1}, 1-\bar{y}_{1}\right)<A_{3}\left(\underline{x}_{2}, \bar{x}_{2}, 1-\underline{y}_{2}, 1-\bar{y}_{2}\right)$,

iv) $A_{1}\left(\underline{x}_{1}, \bar{x}_{1}, 1-\underline{y}_{1}, 1-\bar{y}_{1}\right)=A_{1}\left(\underline{x}_{2}, \bar{x}_{2}, 1-\underline{y}_{2}, 1-\bar{y}_{2}\right)$, $A_{2}\left(\underline{x}_{1}, \bar{x}_{1}, 1-\underline{y}_{1}, 1-\bar{y}_{1}\right)=A_{2}\left(\underline{x}_{2}, \bar{x}_{2}, 1-\bar{y}_{2}, 1-\bar{y}_{2}\right)$, $A_{3}\left(\underline{x}_{1}, \bar{x}_{1}, 1-\underline{y}_{1}, 1-\bar{y}_{1}\right)=A_{3}\left(\underline{x}_{2}, \bar{x}_{2}, 1-\underline{y}_{2}, 1-\bar{y}_{2}\right)$, and $A_{4}\left(\underline{x}_{1}, \bar{x}_{1}, 1-\underline{y}_{1}, 1-\bar{y}_{1}\right) \leq A_{4}\left(\underline{x}_{2}, \bar{x}_{2}, 1-\underline{y}_{2}, 1-\bar{y}_{2}\right)$.

Proof. The linearity is warranted because the equalities only hold if $\left(\underline{x}_{1}, \bar{x}_{1}, 1-\underline{y}_{1}, 1-\bar{y}_{1}\right)=$ $\left(\underline{x}_{2}, \bar{x}_{2}, 1-\underline{y}_{2}, 1-\bar{y}_{2}\right)$.

To check the second condition (that of refining the partial order) in the statement of Def. 4.1, notice that if

$$
\underline{x}_{1} \leq \underline{x}_{2}, \bar{x}_{1} \leq \bar{x}_{2}, \underline{y}_{1} \geq \underline{y}_{2}, \text { and } \bar{y}_{1} \geq \bar{y}_{2} .
$$

then

$$
\underline{x}_{1} \leq \underline{x}_{2}, \bar{x}_{1} \leq \bar{x}_{2}, \quad 1-\underline{y}_{1} \leq 1-\underline{y}_{2}, \text { and } 1-\bar{y}_{1} \leq 1-\bar{y}_{2},
$$

so consequently $A_{i}\left(\underline{x}_{1}, \bar{x}_{1}, 1-\underline{y}_{1}, 1-\bar{y}_{1}\right) \leq A_{i}\left(\underline{x}_{2}, \bar{x}_{2}, 1-\underline{y}_{2}, 1-\bar{y}_{2}\right)$ for all $i \in\{1, \ldots, 4\}$.

From now on we name the order generated by four aggregation functions (as in Prop. 4.2) 4-IVAIF-admissible order.

Remark 5. Given $\mathbf{y} \in L([0,1])$, it follows that $(1-\underline{y}, 1-\bar{y}) \in L^{*}([0,1])$, where

$$
L^{*}([0,1])=\left\{\mathbf{s} \mid \mathbf{s}=\left(s_{1}, s_{2}\right) \text { such that } 0 \leq s_{2} \leq s_{1} \leq 1\right\} .
$$

Then in Prop. 4.2 it is enough that to see, given $\left(\mathbf{p}_{\mathbf{1}}, \mathbf{q}_{\mathbf{1}}\right)=\left(\left[\underline{p}_{1}, \bar{p}_{1}\right],\left(\underline{q}_{1}, \bar{q}_{1}\right)\right),\left(\mathbf{p}_{\mathbf{2}}, \mathbf{q}_{\mathbf{2}}\right)=\left(\left[\underline{p}_{2}, \bar{p}_{2}\right],\left(\underline{q}_{2}, \bar{q}_{2}\right)\right) \in$ $L([0,1]) \times L^{*}([0,1])$, the equalities $A_{i}\left(\underline{p}_{1}, \bar{p}_{1}, \underline{q}_{1}, \bar{q}_{1}\right)=A_{i}\left(\underline{p}_{2}, \bar{p}_{2}, \underline{q}_{2}, \bar{q}_{2}\right)$ hold if and only if $\left(\mathbf{p}_{\mathbf{1}}, \mathbf{q}_{\mathbf{1}}\right)=$ $\left(\mathbf{p}_{2}, \mathbf{q}_{2}\right)$.

However, in order to simplify notation we have imposed a slightly stronger restriction. Anyway, all the given examples in Section 3 satisfy it.

Let a tuple $A=\left\langle A_{1}, \ldots, A_{4}\right\rangle$ of aggregation functions generate an admissible order. Let $B_{i}$ : $[0,1]^{2} \longrightarrow[0,1]$ be four aggregations such that

- $A_{i}(\underline{x}, \bar{x}, \underline{y}, \bar{y})=B_{i}(\underline{x}, \bar{x})$ for $i \in\{1,2\}$, and

- $A_{j}(\underline{x}, \bar{x}, \underline{y}, \bar{y})=B_{j}(\underline{y}, \bar{y})$ for $j \in\{3,4\}$, 
Then the orders $\preceq_{A}^{I V}$ and $\preceq_{B}^{I V}$ may be different. To guarantee that they are actually different it is enough that $B_{3}\left(\underline{y}_{1}, \bar{y}_{1}\right)<B_{3}\left(\underline{y}_{2}, \bar{y}_{2}\right)$ and simultaneously $B_{3}\left(1-\underline{y}_{1}, 1-\bar{y}_{1}\right)>B_{3}\left(1-\underline{y}_{2}, 1-\bar{y}_{2}\right)$ hold true for some $\mathbf{y}_{1}, \mathbf{y}_{2} \in L([0,1])$.

For instance, let $B_{3}(\underline{y}, \bar{y})=\underline{y} \bar{y}$. Here, we have that for $\mathbf{y}_{1}=[0.2,0.2]$ and $\mathbf{y}_{2}=[0.1,0.9]$

$$
\begin{aligned}
& B_{3}(0.2,0.2)=0.04<0.09=B_{3}(0.1,0.9) \\
& B_{3}(0.8,0.8)=0.64>0.09=B_{3}(0.9,0.1) .
\end{aligned}
$$

Proposition 4.3. Let $\alpha_{1}, \alpha_{2}, \alpha_{3}, \alpha_{4} \in[0,1]$, with $\alpha_{1} \neq \alpha_{2}$ and $\alpha_{3} \neq \alpha_{4}$. If

- $A_{i}(\underline{x}, \bar{x}, \underline{y}, \bar{y})=K_{\alpha_{i}}(\underline{x}, \bar{x})$ for $i \in\{1,2\}$, and

- $A_{j}(\underline{x}, \bar{x}, \underline{y}, \bar{y})=K_{\alpha_{j}}(\underline{y}, \bar{y})$ for $j \in\{3,4\}$,

then the tuple $A=\left\langle A_{1}, \ldots, A_{4}\right\rangle$ generates a 4-IVAIF admissible order that is equal to $\preceq_{B}^{I V}$ being $B=<\mathbb{K}_{\alpha_{1}}, \mathbb{K}_{\alpha_{2}}, \mathbb{K}_{\alpha_{3}}, \mathbb{K}_{\alpha_{4}}>$.

Proof. The fact that the aggregation functions satisfy the conditions to generate a 4-IVAIF order is a simple calculation. To prove the equality between the two orders notice that in this case

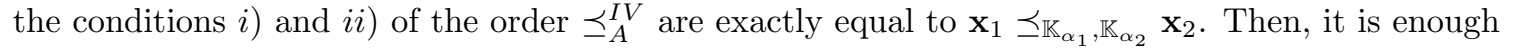
to prove that for all $\gamma$,

$$
\mathbb{K}_{\gamma}\left(1-a_{1}, 1-b_{1}\right)<\mathbb{K}_{\gamma}\left(1-a_{2}, 1-b_{2}\right)
$$

is equivalent to $\mathbb{K}_{\gamma}\left(a_{1}, b_{1}\right)>\mathbb{K}_{\gamma}\left(a_{2}, b_{2}\right)$.

But

$$
\begin{aligned}
\mathbb{K}_{\gamma}\left(1-a_{1}, 1-b_{1}\right) & <\mathbb{K}_{\gamma}\left(1-a_{2}, 1-b_{2}\right) \\
& \Leftrightarrow 1-a_{1}+\gamma\left(1-b_{1}-\left(1-a_{1}\right)\right)<1-a_{2}+\gamma\left(1-b_{2}-\left(1-a_{2}\right)\right) \\
& \Leftrightarrow 1-a_{1}+\gamma\left(a_{1}-b_{1}\right)<1-a_{2}+\gamma\left(a_{2}-b_{2}\right) \\
& \Leftrightarrow a_{2}-\gamma\left(a_{2}-b_{2}\right)<a_{1}-\gamma\left(a_{1}-b_{1}\right) \\
& \Leftrightarrow a_{2}+\gamma\left(b_{2}-a_{2}\right)<a_{1}+\gamma\left(b_{1}-a_{1}\right) \\
& \Leftrightarrow \mathbb{K}_{\gamma}\left(a_{2}, b_{2}\right)<\mathbb{K}_{\gamma}\left(a_{1}, b_{1}\right),
\end{aligned}
$$

so the proof is complete.

Example 4.1. Let $\preceq_{A}^{I V}$ be the order generated by $A=<\mathbb{K}_{0.25}, \mathbb{K}_{0.75}, \mathbb{K}_{0.25}, \mathbb{K}_{0.75}>$. Consider the elements $z_{1}=([0.15,0.35],[0.2,0.5])$ and $z_{2}=([0.15,0.35],[0.1,0.9]) \in \mathcal{L}_{I V}([0,1])$. Since their membership degrees are identical we only need to compare their nonmembership degrees.

In fact,

$$
\begin{aligned}
& \mathbb{K}_{0.25}(0.2,0.5)=0.2+0.25 \cdot(0.5-0.2)=0.275<0.3=0.1+0.25 \cdot(0.9-0.1)=\mathbb{K}_{0.25}(0.1,0.9) \\
& \text { and }([0.15,0.35],[0.1,0.9]) \preceq_{A}^{I V}([0.15,0.35],[0.2,0.5]) .
\end{aligned}
$$




\section{Application to Decision Making}

Decision making problems may be summarized as follows. We have a set of $p$ alternatives:

$$
Z=\left\{z_{1}, \cdots, z_{p}\right\}
$$

and a set of $n>2$ experts:

$$
E=\left\{e_{1}, \cdots, e_{n}\right\} .
$$

Each of the latter provides her/his preferences on the former set of alternatives by means of a preference relation in the following way:

$$
r_{e l}=\left(\begin{array}{cccc}
- & r_{(e l) 12} & \cdots & r_{(e l) 1 p} \\
r_{(e l) 21} & - & \cdots & r_{(e l) 2 p} \\
\cdots & \cdots & - & \cdots \\
r_{(e l) p 1} & \cdots & \cdots & -
\end{array}\right) .
$$

Here $r_{(e l) i j}$, with $i \neq j$, expresses to what extent the expert $l$ (with $l \in\{1, \cdots, n\}$ ) prefers the alternative $z_{i}$ over the alternative $z_{j}$.

We must reach a decision of selecting either an alternative or a set of alternatives, which is (are) optimal as regards the experts assessments.

In [20], it is stated that the resolution of a group decision making problem consists of two steps:

(1) Uniform representation of information. In this phase, the heterogeneous information for the problem (the information can be represented by means of preference orderings or utility functions or fuzzy preference relations) is translated into homogeneous information by means of different transformation functions (see [22]).

(2) Application of a selection procedure. This procedure consists of two phases:

(2.1) Aggregation phase. A collective preference structure is built from the set of individual homogeneous preference structures.

(2.2) Exploitation phase. A given method is applied to the collective preference structure to obtain a selection of alternatives.

We use the theoretical developments in previous sections in the exploitation phase of the group decision making problem considered by Nayagam [23]. In particular, we consider the adaptation of this problem done by Zhang et al in [24]. In this adptation, authors consider that there exists a panel with four possible alternatives for investment:

(1) $z_{1}$ is a car company,

(2) $z_{2}$ is a food company,

(3) $z_{3}$ is a computer company,

(4) $z_{4}$ is an arms company. 
It is necessary to choose the best company for investment.

Let the data in [24] be our collective preference matrix. In the exploitation phase we use the voting method which consists in aggregating the values in each row of the collective matrix $R_{c}$ in such a way that, at the end, we have as many values (pairs of intervals) as rows. Since these latter values are not comparable through the partial order, we will select the alternative associated to the largest pair, according to a considered linear order.

$$
R_{c}=\left(\begin{array}{cccc}
- & ([0.4,0.5],[0.3,0.4]) & ([0.4,0.6],[0.2,0.4]) & ([0.1,0.3],[0.5,0.6]) \\
([0.6,0.7],[0.2,0.3]) & - & ([0.6,0.7],[0.2,0.3]) & ([0.4,0.8],[0.1,0.2]) \\
([0.3,0.6],[0.3,0.4]) & ([0.5,0.6],[0.3,0.4]) & - & ([0.4,0.5],[0.1,0.3]) \\
([0.7,0.8],[0.1,0.2]) & ([0.6,0.7],[0.1,0.3]) & ([0.3,0.4],[0.1,0.2]) & -
\end{array}\right)
$$

To aggregate the values of each row of $R_{c}$ we use the concept of interval-valued intuitionistic t-norms.

Definition 5.1. A mapping $\mathbb{T}:\left(\mathcal{L}_{I V}([0,1])\right)^{2} \rightarrow \mathcal{L}_{I V}([0,1])$ is an interval-valued intuitionistic $t$ norm if it is symmetric, associative, increasing with respect to the partial order $\preceq$ given by Atanassov (also called monotone) and $\mathbb{T}((\mathbf{x}, \mathbf{y}),(\mathbf{1}, \mathbf{0}))=(\mathbf{x}, \mathbf{y})$.

It is easy to see that, if we take the classical product t-norm, $T_{P}(x, y)=x \cdot y$, and its dual t-conorm with respect to the standard negation, $S_{P}(x, y)=x+y-x \cdot y$, the following expression is an interval-valued inuitionistic t-norm: $\mathbb{T}((\mathbf{x}, \mathbf{y}),(\mathbf{z}, \mathbf{t}))=([\underline{x} \cdot \underline{z}, \bar{x} \cdot \bar{z}],[\underline{y}+\underline{t}-\underline{y} \cdot \underline{t}, \bar{y}+\bar{t}-\bar{y} \cdot \bar{t}])$.

Applying $\mathbb{T}$ to each row of $R_{c}$ we get a new matrix, say $R g$, given by:

$$
R g=\left(\begin{array}{c}
z_{1}=([0.016,0.090],[0.720,0.856]) \\
z_{2}=([0.144,0.392],[0.424,0.608]) \\
z_{3}=([0.060,0.180],[0.559,0.748]) \\
z_{4}=([0.126,0.224],[0.271,0.552])
\end{array}\right) .
$$

In this setting, as regards the partial order $\preceq$, it follows

$$
z_{1} \preceq z_{3} \preceq z_{2} \text { and } z_{1} \preceq z_{3} \preceq z_{4}
$$

but $z_{2}$ and $z_{4}$ are not comparable.

For this reason we consider the 4-IVAIF-admissible order $\preceq_{A}^{I V}$ defined through the following aggregation functions.

- $A_{1}\left(\underline{x}_{1}, \bar{x}_{1}, \underline{y}_{1}, \bar{y}_{1}\right)=\frac{2}{20} \underline{x}_{1}+\frac{2}{20} \bar{x}_{1}+\frac{8}{20} \underline{y}_{1}+\frac{8}{20} \bar{y}_{1}$

- $A_{2}\left(\underline{x}_{1}, \bar{x}_{1}, \underline{y}_{1}, \bar{y}_{1}\right)=\frac{10}{20} \underline{x}_{1}+\frac{5}{20} \bar{x}_{1}+\frac{3}{20} \underline{y}_{1}+\frac{2}{20} \bar{y}_{1}$

- $A_{3}\left(\underline{x}_{1}, \bar{x}_{1}, \underline{y}_{1}, \bar{y}_{1}\right)=\frac{1}{20} \underline{x}_{1}+\frac{10}{20} \bar{x}_{1}+\frac{8}{20} \underline{y}_{1}+\frac{1}{20} \bar{y}_{1}$

- $A_{4}\left(\underline{x}_{1}, \bar{x}_{1}, \underline{y}_{1}, \bar{y}_{1}\right)=\frac{1}{4} \underline{x}_{1}+\frac{1}{4} \bar{x}_{1}+\frac{1}{4} \underline{y}_{1}+\frac{1}{4} \bar{y}_{1}$. 
With this order, we have $z_{1} \preceq_{A}^{I V} z_{3} \preceq_{A}^{I V} z_{2} \preceq_{A}^{I V} z_{4}$ and the selected firm is arms company.

However, as it happened in [18] different 4-IVAIFS-admissible orders can lead to different rankings and hence the selection of the best alternative for a given decision making problem can be forced. For instance, in our case if we take $\preceq_{A}^{I V}$ with $A=<\mathbb{K}_{0.25}, \mathbb{K}_{0.75}, \mathbb{K}_{0.25}, \mathbb{K}_{0.75}>$ it comes out that the best alternative is the second one, since $z_{1} \preceq_{A}^{I V} z_{3} \preceq_{A}^{I V} z_{4} \preceq_{A}^{I V} z_{2}$. Nevertheless, for the order $\left(\left[\underline{x}_{1}, \bar{x}_{1}\right],\left[\underline{y}_{1}, \bar{y}_{1}\right]\right) \preceq_{A}^{I V}\left(\left[\underline{x}_{2}, \bar{x}_{2}\right],\left[\underline{y}_{2}, \bar{y}_{2}\right]\right)$ if and only if

- $\left(\bar{x}_{1}<\bar{x}_{2}\right)$, or

- $\left(\bar{x}_{1}=\bar{x}_{2}\right.$ and $\left.\underline{x}_{1}<\underline{x}_{2}\right)$, or

- $\left(\bar{x}_{1}=\bar{x}_{2}, \underline{x}_{1}=\underline{x}_{2}\right.$ and $\left.\bar{y}_{1}>\bar{y}_{2}\right)$, or else

- $\left(\bar{x}_{1}=\bar{x}_{2}, \underline{x}_{1}=\underline{x}_{2}, \bar{y}_{1}=\bar{y}_{2}\right.$ and $\left.\underline{y}_{1} \geq \underline{y}_{2}\right)$

we have that $z_{1} \preceq_{A}^{I V} z_{3} \preceq_{A}^{I V} z_{4} \preceq_{A}^{I V} z_{2}$ and the best alternative is the second one.

To cope with this situation the following algorithm takes different 4-IVAIFS-admissible orders into account simultaneously.

(1) To select several linear orders built with the methods developed in the previous sections.

(2) For each order, to apply in the exploitation phase the voting method with the same aggregations. For instance, $\mathbb{T}=\left(T_{P}, S_{P}\right)$.

(3) To select the alternative which appears as the best placed in the majority of all the so-obtained rankings.

In our considered problem, the chosen alternative through this algorithm is the second one. That is, we must invest our money in a food company. Clearly, the nature of the problem will impose the number of linear orders to be considered and/or the conditions that will force us to use alternative methods.

\section{Conclusions}

In this work we have studied how to construct linear orders between pairs of intervals on $L([0,1])$ that can be used to construct linear orders in Atanassov interval-valued intuitionistic fuzzy sets. We have applied this operator to group decision making problems giving two algorithms, the first one for a particular linear order and the second one which mixes different linear orders.

As a possible development for future research, somewhat related to the main ideas introduced throughout the present manuscript, we point out the introduction of different orderings on families of intervals of the real line could be also analyzed from the point of view of extensions of the canonical ordering of the real line to a superset (namely, $L([0,1]$ ) ) following a suitable set of criteria established a priori. The real line can be immediately embedded into $L([0,1])$ by just considering each real number $\mathrm{x}$ as the degenerate interval $[x, x]$ ).

A similar typical problem corresponds to the extension of linear orders from a finite set to its power set. Indeed, although it is always possible to extend a linear order from a given finite set $U$ to its power set, a typical question that gave rise to some classical papers from the 1970's on (see e.g. [25-28]), is whether or not it is possible to perform an extension that follows a list of criteria imposed a priori. Sometimes, the extension is not possible because the criteria used are, so-to-say, 
contradictory. But, in addition, there are other situations in which the extension is not possible because of a combinatorial explosion which, due to the bigger cardinality of the power set of U, does not leave room to rank all the terms of the power set in an extended linear order, accomplishing all the criteria. Perhaps the most famous result in this direction is the so-called Kannai - Peleg impossibility theorem (see [26]).

However, when the extension does not affect to the whole power set, but to some suitable superset (smaller than the power set), perhaps it may still happen that an extension accomplishing aprioristic criteria is possible, after all. As far as we know, an analysis of this kind where we start with the canonical order of the real line (instead of a linear order on a finite set), and try to extend it to the set of closed intervals of real numbers, following some list of criteria that have been set beforehand, is an open problem.

We leave for future works the interpretation of the length of the intervals in a given decision making problem and its relation with ignorance functions and possibility theory.

\section{Acknowledgment}

The work has been supported by projects TIN2013-40765-P and MTM2012-37894-C02-02 of the Spanish Ministry of Science and the Research Services of the Universidad Publica de Navarra. The last two authors acknowledge the support of the grants VEGA 1/0419/13 and VEGA 1/0420/15.

\section{Bibliography}

[1] E. Barrenechea, J. Fernandez, M. Pagola, F. Chiclana, H. Bustince, Construction of intervalvalued fuzzy preference relations from ignorance functions and fuzzy preference relations. Application to decision making, Knowl.-Based Syst. 58 (2014) 33-44.

[2] H. Bustince, E. Barrenechea, M. Pagola, J. Fernandez, Z.S. Xu, B. Bedregal, J. Montero, H. Hagras, F. Herrera, B. De Baets, A historical account of types of fuzzy sets and their relationships, IEEE T. on Fuzzy Syst. (2015): In press.

[3] J. Sanz, M. Galar, A. Jurio, A. Brugos, M. Pagola, H. Bustince, Medical diagnosis of cardiovascular diseases using an interval-valued fuzzy rule-based classification system, Appl. Soft Comput. 20 (2014) 103-111.

[4] H. Bustince, E. Barrenechea, M. Pagola, Generation of interval-valued fuzzy and Atanassov intuitionistic fuzzy connectives from fuzzy connectives and from $K_{\alpha}$ operators: Laws for conjunctions and disjunctions, amplitude, Int. J. Intell. Syst. 23(6) (2008) 680-714.

[5] G. Deschrijver, C. Cornelis, E.E. Kerre, On the representation of intuitionistic fuzzy t-norms and t-conorms, IEEE T. Fuzzy Syst. 12(1) (2004) 45-61.

[6] K.T. Atanassov, Intuitionistic fuzzy sets, Fuzzy Set Syst. 20(1) (1986) 87-96.

[7] K.T. Atanassov, G. Gargov, Interval-valued intuitionistic fuzzy sets, Fuzzy Set Syst. 31(3) (1989) 343-349. 
[8] H. Bustince, M. Pagola, E. Barrenechea, J. Fernandez, P. Melo-Pinto, P. Couto, H.R. Tizhoosh, J. Montero, Ignorance functions. An application to the calculation of the threshold in prostate ultrasound images, Fuzzy Set Syst. 161(1) (2010) 20-36.

[9] T. Calvo, A. Kolesárová, M. Komorníková, R. Mesiar, Aggregation Operators: properties, Classes and Construction Methods, in: T. Calvo, A. Kolesárová, M. Komorníková, R. Mesiar, Aggregation operators, Studies in Fuzziness and Soft Computing, 97, Physica-verlag HD, 2002, pp.3-104.

[10] G. Beliakov, H. Bustince, D.P. Goswami, U.K. Mukherjee, N.R. Pal, On averaging operators for Atanassov's intuitionistic fuzzy sets, Inform. Sciences, 181(6) (2011) 1116-1124.

[11] G. Beliakov, A. Pradera, T. Calvo, Aggregation Functions: A Guide for Practitioners, Studies in Fuzziness and Soft Computing, 221, Springer, 2007.

[12] G. Birkhoff, Lattice Theory, American Mathematical Society, Colloquium Publications, 25, 1967.

[13] B.A. Davey, H.A. Priestley, Introduction to Lattices and Order, second ed.,Cambridge University Press, Cambridge, 2002.

[14] M.J. Campión, J.C. Candeal, E. Induráin, Representability of binary relations through fuzzy numbers, Fuzzy Set Syst. 157(1) (2006) 1-19.

[15] J.C. Candeal, J. Gutiérrez García, E. Induráin, Universal codomains to represent interval orders, Int. J. Uncertain Fuzz. 17(2) (2009) 197-219.

[16] G. Bosi, J. Gutiérrez García, E. Induráin, Unified representability of total preorders and interval orders through a single function: the lattice approach, Order 26(3) (2009) 255-275.

[17] G. Bosi, A numerical representation of semiorders on a countable set, Riv. di Matematica per le Scienze Economiche e Sociali, 16(2) (1993) 15-19.

[18] H. Bustince, J. Fernandez, A. Kolesárová, R. Mesiar, Generation of linear orders for intervals by means of aggregation functions, Fuzzy Set Syst. 220(2013) 69-77.

[19] M. Komorníková and R. Mesiar, Aggregation functions on bounded partially ordered sets and their classification, Fuzzy Set Syst. 175(1) (2011) 48-56.

[20] J. Fodor, M. Roubens, Fuzzy preference modelling and multicriteria decision support, In Theory and Decision Library, Kluwer Academic Publishers, 1994.

[21] M. Grabisch, J.L. Marichal, R. Mesiar, E. Pap. Aggregation Functions, Cambridge University Press, Cambridge, 2009.

[22] F. Chiclana, F. Herrera, E. Herrera-Viedma, Integrating three representation models in fuzzy multipurpose decision making based on fuzzy preference relations, Fuzzy Set Syst. 97(1) (1998) $33-48$.

[23] V.L.G. Nayagam, S. Muralikrishnan, G. Sivaramanr, Multi-criteria decision-making method based on interval-valued intuitionistic fuzzy sets, Expert Syst. Appl. 38 (2011) 464-467. 
[24] Q. Zhang, H. Xing, F. Liu, J. Ye, P. Tang, Some new entropy measures for interval-valued intuitionistic fuzzy sets based on distances and their relationships with similarity and inclusion measures, Inform. Sciences, 283 (2014) 55-69.

[25] P. Gärdenfors, Manipulation of social choice functions, J. of Economic Theory, 13(2) (1976) 217-228.

[26] Y. Kannai, B. Peleg, A note on the extension of an order on a set to the power set, J. of Economic Theory, 32(1) (1984) 172-175.

[27] P.C. Fishburn, Comment on the Kannai-Peleg impossibility theorem for extending orders, J. of Economic Theory, 32(1) (1984) 176-179.

[28] W. Bossert, On the extension of preferences over a set to the power set: An axiomatic characterization of a quasi-ordering, J. of Economic Theory, 49(1) (1989) 84-92. 